\title{
Erinnerung an Alexander Tschirch (1856-1939), Bern
}

\author{
Von Gottfried Schramm
}

Die wissenschaftliche Entwicklung der Pharmakognosie und Pharmazie ist weltweit und unabdingbar verbunden mit der Lehre und Forschung des 1856 zu Guben in der preußischen Niederlausitz geborenen, seit 1890 an der Universität Bern wirkenden Pharmakognosten und Pharmazieprofessors Alexander Tschirch, dessen Geburtstag sich am 17.Oktober 1986 zum 130. Male jährte.

Bereits 1915 erschienen seine «Vorträge und Reden», 1921 seine Lebenserinnerungen «Erlebtes und Erstrebtes», wenig später «Fünfzig Jahre im Dienste der Pharmacie und Naturforschung (1872-1922)» und 1925/26 «Kongreßfahrten», die als Drucke grundlegende Teilaspekte seiner Arbeiten widerspiegeln.

Im Sinne einer Dokumentation pharmaziehistorisch bedeutsamer Autographen sei es gestattet, nachfolgend eine eindrucksvolle Glückwunschadresse des Berner Apothekers Bernhard Studer vom 17.Oktober 1931 wiederzugeben, die das vorhandene Schrifttum gleichsam ergänzt. Der Briefschreiber, ein renommierter Berner Standesvertreter, Schüler Alexander Tschirchs und nahestehender Freund der Familie, überliefert und zeichnet in liebevoller Verbundenheit zu seinem Lehrer ein zeitgenössisches Bild des Jubilars in Würdigung dessen Verdienste um die Pharmazie, das Zeiten überdauerte.

Lieber Herr Professor!

Gestatten Sie Ihrem alten + dankbaren schüler, sich den vielen gratulanten anzuschließen, die sich heute bei anlaß Ihres 76. geburtstages bei Ihnen melden, um Ihnen Ihre wünsche für Ihr ferneres wohlergehen + ihre dankbarkeit auszudrücken für all das, was Sie in langen jahren harter arbeit für die pharmacie geleistet haben.

Ich freue mich mit allen anderen ganz besonders, daß es Ihnen vergönnt war, Ihr lebenswerk durch die erbauung des neuen pharmazeutischen institutes zu krönen.

Als berner apotheker danke ich Ihnen für die treue die Sie unserem stande durch dick und dünn gehalten haben + ganz speziell dafür, daß Sie trotz Ihres hohen alters + ungeachtet Ihrer zeitweise geschwächten gesundheit, sich der großen mühe unterzogen haben, der berner 
regierung eine unserem stande würdige bildungsanstalt abzuringen, eine anstalt, die als mustergültig den blick der internationalen pharmacie nach bern ziehen wird.

Seitdem wir Thr 70.jahr feiern durften, sind Sie wohl an jahren älter, geistig + körperlich aber jünger geworden. Ich beglückwünsche Sie + Ihre verehrte familie für die art wie Sie das problem des alterns lösen + ich hoffe sehr, Sie werden uns (ich rechne mich auch etwas zu Ihrer Familie) noch recht lange so jugendlich + arbeitsfähig bleiben.

Ich bin Ihr stets in zuneigung ergebener

Bernhard Studer

Eine lebensnahe, liebenswürdige Schilderung «Prof.dr. Tschirch, Bern, 75 Jahre alt» veröffentlichte Bernhard Studer, Bern, auf Wunsch der Redaktion der Estländischen Apotheker-Zeitung in «Eesti Rohuteadlane» VI (1931), 231-234.

\section{Summary}

Professor Alexander Tschirch (1856-1939), Bern, was a highly distinguished and much admired professor of pharmacognosy and pharmaceutical chemistry of the University of Bern.

This paper pays homage to him on the 130th anniversary of his birth. It consists mainly of a letter (dated Oct.17, 1931) by Dr. Bernhard Studer, Bern, portraying Professor Tschirch as an ideal teacher, a hard worker and the founder of the modern pharmaceutical institute of the university of Bern, against the academic background in which he moved, when he passed the three-quarters century mark.

Dr.sc.nat. Dr.phil. Gottfried Schramm

Waidspital, Tièchestraße 99

CH-8037 Zürich 